\title{
Publisher Correction: Whole-tissue biopsy phenotyping of three-dimensional tumours reveals patterns of cancer heterogeneity
}

Nobuyuki Tanaka, Shigeaki Kanatani, Raju Tomer, Cecilia Sahlgren, Pauliina Kronqvist, Dagmara Kaczynska, Lauri Louhivuori, Lorand Kis, Claes Lindh, Przemysław Mitura, Andrzej Stepulak, Sara Corvigno, Johan Hartman, Patrick Micke, Artur Mezheyeuski, Carina Strell, Joseph W. Carlson, Carlos Fernández Moro, Hanna Dahlstrand, Arne Östman, Kazuhiro Matsumoto, Peter Wiklund, Mototsugu Oya, Ayako Miyakawa, Karl Deisseroth and Per Uhlén

Correction to: Nature Biomedical Engineering https://doi.org/10.1038/s41551-017-0139-0; published online 2 October 2017.

In this Article originally published, owing to a technical error, author affiliations were incorrectly assigned in the HTML version; the PDF was correct. These errors have now been corrected.

Published online: 2 January 2018

https://doi.org/10.1038/s41551-017-0162-1 